(2 of 2) 2001;57:1383-1388). (Reprints: Dr R Kurlan, Department of Neurology, University of Rochester School of Medicine and Dentistry, 601 Elmwood Ave, Box 673, Rochester, NY 14642).

COMMENT. This represents a further publication in a long series of studies of tic disorders and Tourette syndrome conducted at the University of Rochester and involving school children in Monroe County. The observed prevalence of tics among children attending regular classes in the present study (18.5\%) is higher than that previously quoted in the literature (4 to 16\%), but the prevalence (27\%) among children in special education is similar (26\%) to a study cited by Robertson MM (Brain 2000;123:425-462). Comparing a group of 35 children in special education with 35 in regular classes, the incidence of tics was $26 \%$ and $6 \%$, respectively. Although the authors of the Rochester study state that only a small number of subjects took medication on the day of the interview, it is noteworthy that $30 \%$ of SpEd students were under treatment with stimulant medication and presumably were diagnosed with ADHD. Despite recent studies that minimize an association (Law SF, Schachar RJ. LAm Acad Child Adolesc Psvchiatry 1999;38:944951; Ped Neur Briefs Aug 1999;13:57-58), our experience and earlier studies have shown that stimulant therapy for ADHD may precipitate tics in susceptible children, and the effect is dose-related (Borcherding BG et al. Psychiatry Res 1990;33:83-94; Tanner CM, Goldman SM. Neurol Clin 1997;15:395-402). Furthermore, tics and Tourette syndrome received no or only passing mention in neurological literature and textbooks until the use of stimulants became popular in the 1960s. (Millichap JG. Tics and Tourette syndrome. In Attention Deficit Hyperactivity and Learning Disabilities. PNB Publishers, 2001). It is prudent to monitor for tics during treatment of ADHD with stimulants.

\title{
HYPOTHYROIDISM, MOTOR CLUMSINESS, AND ADHD
}

The behavioral characteristics of 63 children with early-treated congenital hypothyroidism $(\mathrm{CH})$ were evaluated by parent and teacher ratings and compared to 34 healthy controls (ages 7.5-9.5 yrs) at the University of Jyvaskyla, Finland. Children with $\mathrm{CH}$, especially those with thyroid agenesis, were more introverted, insecure, and isolated, and showed more motor clumsiness, rather than social negativity and inattention. Children with thyroid agenesis were clumsier than those with thyroid dysgenesis. It is suggested that the motor problems contribute to the introverted behavior. The more severe the thyroid deficiency, the greater probability for behavior problems. The lack of effect on attention challenges claims of an association between $\mathrm{ADHD}$ and thyroid dysfunction. (Kooistra L, Stemerdink N, van der Meere J, et al. Behavioral correlates of early-treated congenital hypothyroidism. Acta Paediatr Oct 2001;90:1141-1146). (Respond: Dr Libbe Kooistra, Research Institute for Olympic Sports, University of Jyvaskyla, University Campus, Rautpohjankatu 6, FIN-40700 Jyvaskyla, Finland).

COMMENT. Early-treated congenital hypothyroidism is associated with introverted behavior and motor clumsiness, and not inattention, impulsivity, and hyperactivity, the core symptoms of ADHD.

\section{SEIZURE DISORDERS}

\section{VIDEO-EEG IN INFANTILE SPASMS}

Early electroclinical manifestations and treatment responses were evaluated by video-EEG in 44 infants with infantile spasms. Mean ages at onset and at first video-EEG were 5.3 and 5.9 months, respectively. Etiology was cryptogenic 
in 13 and symptomatic in 31 infants. Vigabatrin was the initial treatment in 36 , and all infants were followed until 12 months of age. On the first video-EEG before treatment, 27 infants showed hypsarrhythmia, and 17 had multifocal spikes with normal EEG background. A 4-hour recording including waking, sleep, and awakening was usually sufficient to detect spasms. Subtle, asymmetric or asynchronous spasms, and asymmetric ictal or interictal EEG abnormalities were associated with symptomatic etiology and poor cognitive and seizure outcome. Treatment modifies both spasms and EEG; spasms may become subtle and only discernible on EEG, and hypsarrhythmia may be replaced by multifocal spikes. Hypasarrhythmia rarely persists following vigabatrin treatment. Complete eradication of spasms, including subtle variants, is essential for a favorable cognitive outcome. (Gaily E, Liukkonen E, Paetau R et al. Infantile spasms: diagnosis and assessment of treatment response by video-EEG. Dev Med Child Neurol Oct 2001;43:658-667). (Respond: Dr Eija Gaily, PO Box 280, Hospital for Children and Adolescents, FIN-00029 HYKS, Finland).

COMMENT. Video-EEG may be essential for the initial diagnosis of infantile spasms, especially in infants with symptomatic etiology and subtle spasms. It is also necessary in assessing response to treatment and the exclusion of persisting subtle spasms not clinically evident.

\section{RISK FACTORS FOR EARLY NEONATAL SEIZURES}

Prenatal and perinatal risk factors for neonatal seizures occurring in 100 newborns in the first week were determined in a case-control study at Grottaferrata, the University of Rome, and other centers, Italy. The majority (71\%) had more than one type of seizure, the most common being generalized tonic (29\% of infants). Less frequently, multifocal clonic (13\%), focal clonic (9\%), and myoclonic seizures (5\%); all were associated with subtle seizures. Interictal EEG was normal in 20\%, and showed asymmetries of background activity in $47 \%$, burst suppression in 5\%, and episodic voltage attenuation in $28 \%$. Etiologies presumed to be responsible for seizures included hypoxic-ischemic encephalopathy in $30 \%$, intracranial hemorrhage (10\%), hypocalcemia (9\%), meningitis (8\%), hypoglycemia (2\%), and unknown (10\%). A history of epilepsy in first degree relatives was found in 4 cases and for none of the controls. Parents of cases had a lower socioeconomic status than those of controls. Neonatal seizures were associated with maternal disease in the 2 years preceding the pregnancy, excessive weight gain, placental pathology, preeclampsia, low geastational age, low birth weight, and neonatal jaundice. Cardiopulmonary resuscitation was needed in $37 \%$ of cases and none of the controls. (Arpino C, Domizio S, Carrieri MP et al. Prenatal and perinatal determinants of neonatal seizures occurring in the first week of life. I Child Neurol October 2001;16:651-656). (Respond: Dr Carla Arpino, E Litta Rehabilitation Center for Developmental Disabilities, Via Anagnina Nuova, 13, 00046 Grottaferrata, Rome, Italy).

COMMENT. Neonatal seizures in the first week are strongly associated with low gestational age and low birthweight, risk factors that might be preventable. Other familial. maternal, and neonatal factors are associated, including placental pathology, preeclampsia, and a family history of epilepsy and febrile seizures.

\section{KETOGENIC DIET IN REFRACTORY FOCAL SEIZURES}

The efficacy of the ketogenic diet in the treatment of intractable focal seizures was studied retrospectively in 34 patients (mean age, $7.5 \mathrm{yrs}$; range, 4 months to $29 \mathrm{yrs}$ ) and compared to 100 patients with generalized seizures at the 